\title{
Agewise Variation of Intraocular Pressure, Pachymetryand Keratometry in the Urban Areas of Assam
}

\author{
JuriPathak
}

Abstract: Purpose:The study is undertaken keeping in mind the following objectives by considering 7 age groups between the age of 5 to 40 years:

- To determine the Normal Frequency Distribution of Intraocular Pressure (IOP), Axial Length and Pachymetry for both eyes.

- $\quad$ To find the Distribution of Pachymetry, IOP and Axial lengthwith Age for each age group.

- To analyze the variance between Pachymetry, Intraocular Pressure and Axial Length for both eyes independently.

- $\quad$ To study the multiple regression between Pachymetry, Intraocular Pressure and Axial Length.

Methods:Apopulation of 100 subjects, 200 eyes were enrolled in this study. All patients were selected from the outpatient clinic of a Private Nursing home.Patient age ranged from 5 to 40 years. Records of those patients who have gone under ophthalmic examination to measure IOP, central Pachymetry, Kerotometry and Axial length are collected.Analysis is done with the help of statistical software SPSS V.15 for windows operating system.

Results:Study found a statistically significant relation between the IOP and the corneal curvature both in the correlation test and in the multivariate study. From the correlation table it is seen thatPachymetry and I.O.P. for the Right eye is highest for the age group of 31-35 years. Again for the Age group 26-30 years Correlation value between Pachymetry and IOP for the Right eye shows that there is very insignificant relation between them. For the Age group of 36-40 yrs, correlation value between IOP and Axial Length for the Left eye have the highest negative trend. It implies that with increase in IOP, Axial length goes on decreasing and vice versa. Pachymetry significantly affectsIOP. A relation was found between corneal thickness and age by groups.

Conclusion: It is seen thattreatments of Axial Length, I.O.P. and Pachymetry are not alike.The highest treatment mean effects are 23.78 \& 23.82 due to the feedstuff Axial Length.Hence if a choice is to be made among the three treatments Axial Length, I.O.P. and Pachymetry, treatment Axial Length is most effective.

\section{Introduction}

Eyes are the significant human sense organ that detect light, and send signals along the optic nerve to the visual and other areas of the brain. $80 \%$ of the data input to the brain comes from vision. There are different Parameter which may potentially exhibit wide differences within a given population such as Axial Length, Pachymetry, Intraocular Pressure (IOP) etc. The purpose of this work is to assist clinicians with the interpretation of statistics by providing a general understanding of the basic concepts that lead to choosing an appropriate statistical test for common study designs. The choice of sampling design and the tools of analysis were determined based on the specific objectives of the study, hypotheses and the characteristics of the universe of the study. Adequate care was also taken to ensure that the sample includes all kinds of records for different age groups.

For the statistical study of different parameters between different age groups, SPSS 15.0 software for Windows is used. Normality of data in each group was confirmed using normal probability plots. Measurements were recorded as the mean \pm 1 standard deviation (using $\{n-1\} 1 / 2$ in the denominator of the definition for standard deviation, where $\mathrm{n}$ is the number of observations for each measurement). Differences between data sample means were determined by a simple $t$-test and $P$ values less than .05 were considered statistically significant. Correlations between parameters were studied using the Pearson Bivariate correlation test.IOP predictions were assessed via a multiple linear regression analyses with respect to those parameters and the significancelevels were evaluated by the paired, 2-tailed $t$ test.After performing Multiple Linear Regression it is found that IOP is a significant predictor of Axial length for both the eyes. Pachymetry and Keratometryare excluded from the list.

\section{Analysis of Variance (ANOVA)}

II. Results

It is found that tabulated value of $\mathrm{F}$ is 2,297 (Table-I) at 5\% level of significance is 3.00. From the calculation it is found that Variance is 1343.81 for the left eye and 1723.69 for the right eye which is highly significant than the Tabulated F value. Here the Calculated Critical Difference (C.D.) for the left eye is 4.42 and 
Agewise variation of Intraocular Pressure, PachymetryandKeratometry in the urban areas of Assam for the right eye is 4.41. The treatment mean effects, arranged in descending order of magnitude, are given in the Table-II.Comparing these differences with the Critical Difference (C.D.) it is found that Axial Length differ significantly from each of the treatment I.O.P. and Pachymetry.

Table -I :One way ANOVA between Pachymetry, I.O.P. and Axial Length for the both left and right eyes.

Table-I

\begin{tabular}{|c|c|c|c|c|c|c|c|c|}
\hline \multirow{2}{*}{$\begin{array}{l}\text { Source of } \\
\text { variation }\end{array}$} & \multirow{2}{*}{$\begin{array}{l}\text { df } \\
\text { (Both } \\
\text { eyes) }\end{array}$} & \multicolumn{2}{|c|}{ SS } & \multicolumn{2}{|l|}{ MSS } & \multicolumn{2}{|l|}{ VR(F) } & \multirow{2}{*}{ Inference } \\
\hline & & Left & Right & Left & Right & Left & Right & \\
\hline Treatment & 2 & 25759.67 & 26151.51 & 12879.84 & 13075.76 & 1343.81 & 1723.69 & \multirow{3}{*}{$\begin{array}{l}\text { Highly } \\
\text { significant }\end{array}$} \\
\hline$\overline{\text { Error }}$ & 297 & 2846.62 & 2253.02 & 9.58 & 7.59 & & & \\
\hline Total & 299 & 28606.29 & 28404.53 & & & & & \\
\hline
\end{tabular}

Table - II : The treatment mean effects, arranged in descending order of magnitude

Table - II

\begin{tabular}{|l|l|l|l|l|l|l|}
\hline \multirow{2}{*}{ Treatment } & \multicolumn{4}{|l|}{ Mean gain in weight } & \multicolumn{2}{l|}{ Difference } \\
\cline { 2 - 8 } & Left & Right & Left & Right & Left & Right \\
\hline Axial Length & 23.78 & 23.8223 & & & & \\
\hline I.O.P. (Left) & 12.12 & 12.06 & 11.76 & 11.76 & & \\
\hline Pachymetry(Left) & 0.973195876 & 0.965252525 & 22.91 & 22.86 & 11.15 & 11.095 \\
\hline
\end{tabular}

III.

Normal Probability Plots:

\section{Q-Q plots for Keratometry and Axial Length}

In a perfect normal frequency distribution, the mean, median and mode are equal. The dependent variable(s) is normally distributed. Normal distribution with a Q-Q plot is used to check the dependency.

Axial length predictions were assessed via a multiple linear regression study with respect to those factors showing a significant association in correlation tests: Corneal thickness, corneal curvature and Intraocular pressure.
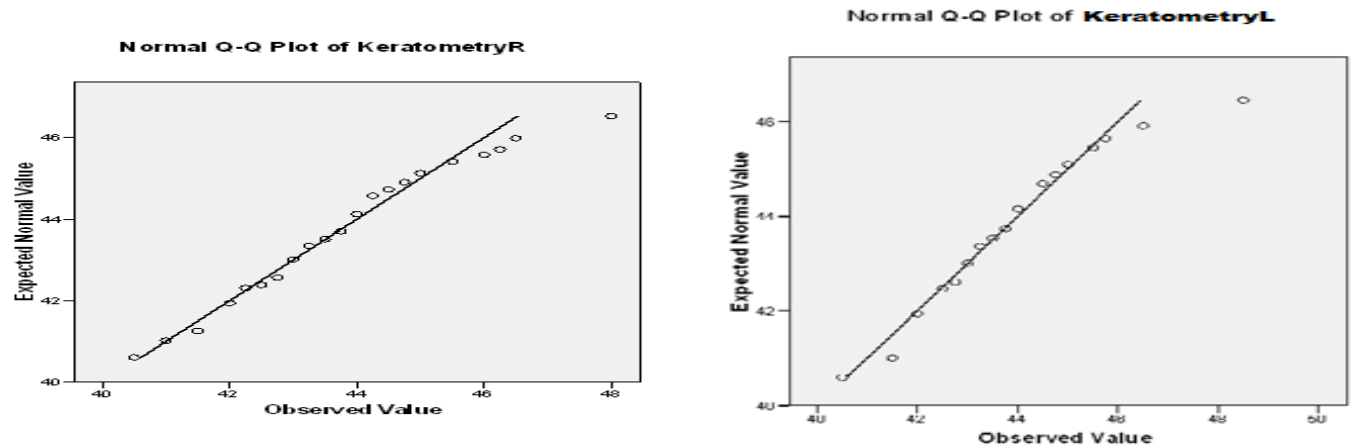

Fig 1: Normal Q-Q plots KeratometryFig 2 :NormalQ-Q plots Keratometry (Right eye)(Left eye)

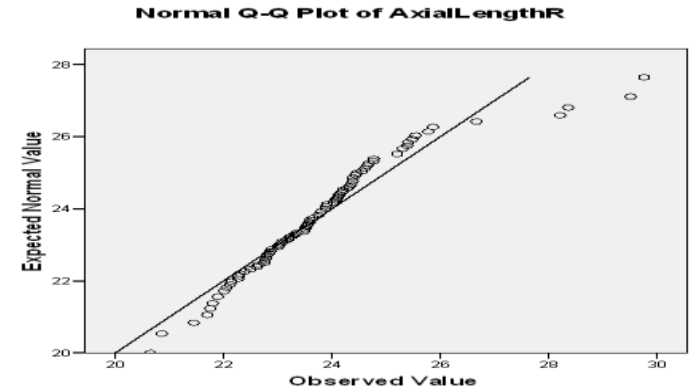

Fig 3: Normal Q-Q plots Axial Length

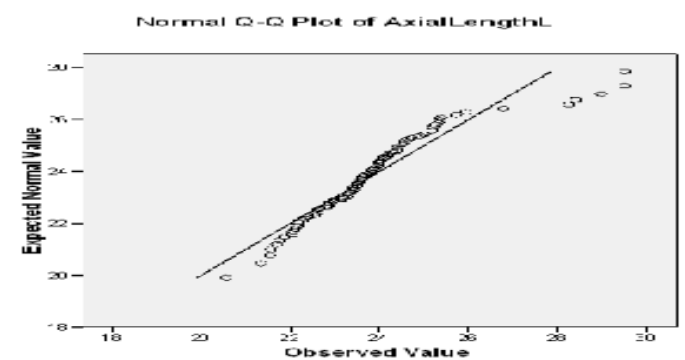

Fig 4:NormalQ-Q plots Axial Length

(Right eye)(Left eye) 
The graph Q-Q plot of normality of each parameter reveals that we should accept the hypothesis of normality, since the points are situated almost along the straight line. Although the normality hypothesis can be accepted, the regression would not be reliable, since the values deviated from the straight line of normality.

\section{Co-relation between parameters of different age groups}

The relationship between the corneal thickness i.e. Pachymetry and the corneal curvature i.e. Keratometry with the IOP has been calculated by stepwise linear regression analyses. Stepwise logistic regression let us evaluate the independent influence of each parameter.Co-relation Table for different age groups is shown in Table-III.

Table-III

\begin{tabular}{|l|l|l|l|l|l|l|}
\hline \multirow{2}{*}{ Age } & \multicolumn{2}{l|}{ IOP and Pachymetry } & \multicolumn{2}{l|}{ IOP and Axial Length } & \multicolumn{2}{l|}{ IOP and Age } \\
\cline { 2 - 7 } & Left & Right & Left & Right & Left & Right \\
\hline $\mathbf{5}-\mathbf{1 0} \mathbf{Y r} \boldsymbol{s}$ & 0.226519 & 0.407916 & 0.28514 & 0.418662 & 0.211553 & 0.316613 \\
\hline $\mathbf{1 1}-\mathbf{1 5} \mathbf{r} \boldsymbol{s}$ & 0.54523 & 0.69278 & 0.587037 & 0.589416 & -0.58632 & -0.59318 \\
\hline $\mathbf{1 6}-\mathbf{2 0} \mathbf{r} \boldsymbol{s}$ & 0.483367 & 0.483825 & 0.43912 & 0.51204 & 0.09303 & 0.048667 \\
\hline $\mathbf{2 1}-\mathbf{2 5} \mathbf{r} \boldsymbol{s}$ & 0.330927 & 0.17413 & 0.273342 & 0.353929 & -0.24308 & -0.0898 \\
\hline $\mathbf{2 6}-\mathbf{3 0} \mathbf{r} \boldsymbol{s}$ & 0.262981 & 0.023199 & -0.17612 & -0.28868 & 0.259615 & 0.27454 \\
\hline $\mathbf{3 1}-\mathbf{3 5} \mathbf{r} \boldsymbol{s}$ & 0.57242 & 0.835745 & 0.612679 & 0.821995 & -0.2809 & 0 \\
\hline $\mathbf{3 6}-\mathbf{4 0} \mathbf{Y} \boldsymbol{r} \mathbf{s}$ & -0.60613 & 0.453014 & -0.69389 & 0.35729 & 0.800641 & -0.33265 \\
\hline
\end{tabular}

From Table-III it is found that for the age group of 31-35 yrs, table value between Pachymetry and I.O.P. (0.835745) for the Right eye is highest. This implies that the rate at which the Pachymetry value increases with increase in I.O.P. is highest than other tabular value.

The tabulated correlation value for the Age group 31-35yrs between I.O.P. and Age for the Right eye is 0, which implies that there is no relation between IOP and Age in case of Right eyes.

For the Age group 26-30 yrs. Correlation value between Pachymetry and IOP for the Right eye is 0.023199 which implies there is very insignificant relation between them.

For the Age group 36-40 yrs, correlation value between IOP and Axial Length for the Left eye ie. 0.69389 have the highest negative trend. It implies that with increase in IOP, Axial length goes on decreasing and vice versa.

\section{References}

Edward A. H. Mallen, PritiKashyap; "Technical Note: Measurement of retinal contour and supine axial length using the Zeiss IOLMaster"; Ophthalmic and Physiological Optics.

[2] Francesco Bandello, Alessandra Tavola, Luisa Pierro, GiulioModorati, Claudio Azzolini, Rosario Brancato; “Axial Length and Refraction in Retinal Vein Occlusions"; Ophthalmologica 1998; V(212); p(133-135).

[3] George Tomais; GerasimosGeorgopoulos; ChryssanthiKoutsandrea; MichalisMoschos; "Correlation of central corneal thickness and axial length to the optic disc and peripapillary atrophy among healthy individuals, glaucoma and ocular hypertension pati ents"; Clinical Ophthalmology 2008:2(4)p(981-988).

[4] Gillis A, ZeyenT;"Comparison of Optical Coherence Reflectometry and Ultrasound Central Corneal Pachymetry"; Bull. Soc. belgeOphtalmol., 292, 2004, p(71-75).

[5] Jacob Cohen ; "Statistical Power Analysis for the Behavioral Sciences"; Edition: 2.

[6] Jose Luis Olivares Jimenez, Juan Jose Serrano Perez, Daniel Serrano Laborday, Enrique HitaVillaverde;"'Statistical study of the ocular parameters of the human eye"; Opt. Pur. Apl. (1997), Vol (30)-n 2.

[7] Julie Pallant; "SPSS Survival Manual: A Step by Step Guide to Data Analysis Using SPSS"; Edition: 3, revised; Published by Allen \&Unwin, 2007.

[8] Jens F. Jordan,Silke Joergens, Sven Dinslage, Thomas S. Dietlein and Gunter K. Krieglstein; "Central and paracentral corneal pachymetry in patients with normal tension glaucoma and ocular hypertension" ; Graefe's Archive for Clinical and Experimental Ophthalmology; Volume 244, 2006; p(177-182)

[9] Kohlhaas M, Boehm AG, Spoerl E, Pursten A, GreinHJ,Pillunat LE. Effect of central corneal thickness, corneal curvature, and axial length on applanationtonometr; Arch Ophthalmol 2006; 124: p(471-476). K. A. Brownlee (1960); "Statistical Theory and Methodology in Science and Engineering", John Wiley \& Sons, Inc., New York, p (236).

[11] Lorri B. Wilson, Graham E. Quinn, Gui-shuang Ying, Ellie L. Francis, GregorSchmid, An Lam, Josh Orlow and Richard A. Stone1; "The Relation of Axial Length and Intraocular Pressure Fluctuations in Human Eyes"; Invest Ophthalmol Vis Sci.,May 2006, Vol. 47, No. 5 .

[12] MPakravan, A Parsa, M Sanagou and C F Parsa; Central corneal thickness and correlation to optic disc size: a potential link for susceptibility to glaucoma; British Journal of Ophthalmology 2006;91:p(26-28).

[13] Mohamed Hosny, Jorge L. Alio, PascualClaramonte,Walid H. Attia, Juan J. Perez-Santonja; "Relationship Between AnteriorChamber Depth, Refractive State,Corneal Diameter, and Axial Length";Journal of Refractive Surgery; December 1999, Volume 16 p(336-340).

[14] Mika Harju;"Exfoliation glaucoma:Studies on intraocular pressure, optic nerve head morphometry,and ocular blood flow"; Department of Ophthalmology University of Helsinki, Helsinki, Finland. 
Agewise variation of Intraocular Pressure, PachymetryandKeratometry in the urban areas of Assam

[15] Massey, F. J. Jr. (1951). The Kolmogorov-Smirnov test of goodness of fit; Journal of the American Statistical Association; Vol. 46; $\mathrm{p}(70)$.

[16] PawelLewicki and Thomas Hill, (2007):"STATISTICS Methods and Applications".

[17] Robert A. Yaffee; "Regression Analysis with SPSS".

[18] Sanchez-Tocino, Bringas-Calvo R, Iglesias-CortinasH;Correlation Between Intraocular Pressure,Paquimetry And Keratometry in A Normal Populatio ; ARCH SOC ESP OFTALMOL 2007; 82: p(267-272).

[19] Singh Ravinder P, Goldberg I, Graham SL, Sharma A, Mohsin M; Central corneal thickness, tonometry and, ocular dimensions in glaucoma and ocular hypertension; J Glaucoma 2001; 10: p(206-210).

[20] S. C. Gupta, V. K. Kapoor; "Fundamentals of applied Statistic"; 3rd Edition.

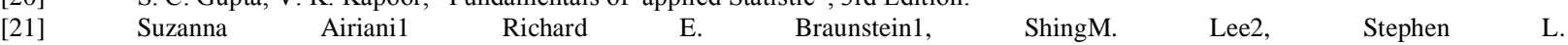
Trokel;"'Evaluating Central Corneal Thickness Measurements with Non-contact Optical Low Coherence Reflectometryand Contact Ultrasound Pachymetry"; Dept. of Ophthalmology, College of Physicians and Surgeons of Columbia University, New York. 